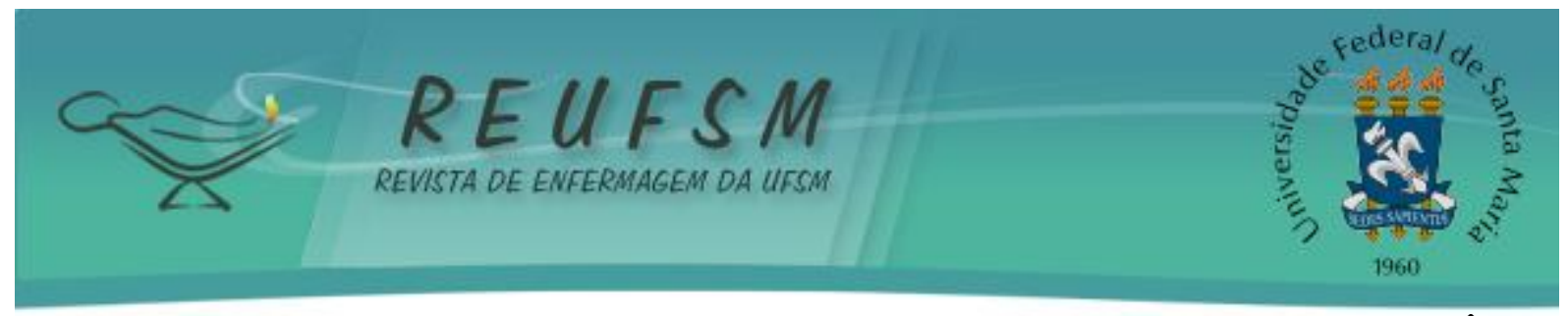

RELATO DE EXPERIÊNCIA

\title{
AÇÕES EDUCATIVAS NA PRÁXIS DO CUIDADO EM DOENÇAS CARDIOVASCULARES: UM RELATO DE EXPERIÊNCIA
}

\author{
EDUCATIVES ACTIONS IN PRAXIS OF THE CARE IN CARDIOVASCULARES DISEASE: A \\ EXPERIENCE REPORT
}

\section{ACCIONES EDUCATIVAS EN LA PRAXIS DEL CUIDADO EN ENFERMEDADES CARDIOVASCULARES: UN INFORME DE EXPERIENCIA}

\author{
Márcia Gabriela Rodrigues de Lima ${ }^{1}$ \\ Elisabeta Albertina Nietsche ${ }^{2}$ \\ Janilene Camara Botega ${ }^{3}$ \\ Cristiane Apio Motta ${ }^{4}$ \\ Glaucia Dal Omo Nicola ${ }^{5}$ \\ Larice Goncalves Terra ${ }^{6}$ \\ Claudiane Botolli ${ }^{7}$
}

RESUMO: Objetivo: descrever as atividades de promoção, prevenção, recuperação e tratamento de doenças cardiovasculares desenvolvidas na prática assistencial, juntamente com os usuários cardiopatas ou portadores de algum fator de risco à doença. Método: trata-se de um relato de experiência obtido em um Laboratório de Hemodinâmica, Unidade de Cardiologia Intensiva e Estratégia de Saúde da Família em setembro de 2009 a julho de 2010, no período de estágio supervisionado no Curso de Graduação em Enfermagem. As ações consistiram em práticas de educação em saúde no cenário hospitalar e comunitário. Resultados: as atividades educativas foram desenvolvidas na admissão do usuário ao serviço, em técnicas grupais e visitas domiciliárias a fim de que os mesmos se co-responsabilizassem pela sua promoção da saúde, prevenção, tratamento e recuperação das doenças cardiovasculares. Conclusão: destaca-se a necessidade de empregar ações de educação em saúde nas práticas assistenciais como elemento emancipatório dos usuários em seu autocuidado.

Descritores: Educação em saúde; Doenças cardiovasculares; Cuidados de enfermagem.

ABSTRACT: Objective: to describe the activities of promotion, prevention, recovery and treatment of cardiovascular diseases developed in care practice, together with the users with heart disease or patients with a risk factor for the disease. Method: it is an experience report developedin Hemodynamic Laboratory, Intensive Care Unit Cardiology

\footnotetext{
${ }^{1}$ Enfermeira. Mestranda do Programa de Pós-Graduação em Enfermagem da Universidade Federal de Santa Maria/RS (PPGEnf./UFSM). Integrante do Grupo de Estudos e Pesquisa em Enfermagem e Saúde (GEPES/UFSM/CNPQ). E-mail: grlmarcia@yahoo.com.br

${ }^{2}$ Enfermeira. Doutora em Enfermagem. Docente do Curso de Graduação em Enfermagem e do PPGEnf. da UFSM. Coordenadora do GEPES/UFSM/CNPQ. E-mail: enietsch@terra.com.br

${ }^{3}$ Graduanda do $7^{\circ}$ semestre do Curso de Enfermagem da UFSM. Integrante do GEPES/UFSM/CNPQ. Email: janicamega@yahoo.com.br

${ }^{4}$ Enfermeira. Aluna do Programa Especial de Graduação de Formação de Professores para Educação Profissional. Integrante do GEPES/UFSM/CNPQ. E-mail: crisapiomotta@hotmail.com

${ }^{5}$ Enfermeira. Mestranda pelo Programa de Pós-Graduação em Enfermagem da Universidade Federal do Rio Grande. Integrante do GEPES/UFSM/CNPQ. E-mail: glaucianicola@hotmail.com

${ }^{6}$ Graduanda do $2^{\circ}$ semestre do Curso de Enfermagem da UFSM. Integrante do GEPES/UFSM/CNPQ. Email: lariceterra@hotmail.com

${ }^{7}$ Enfermeira do Hospital Universitário de Santa Maria no Setor de Hemodinâmica. E-mail: claudianenf@yahoo.com.br
} 


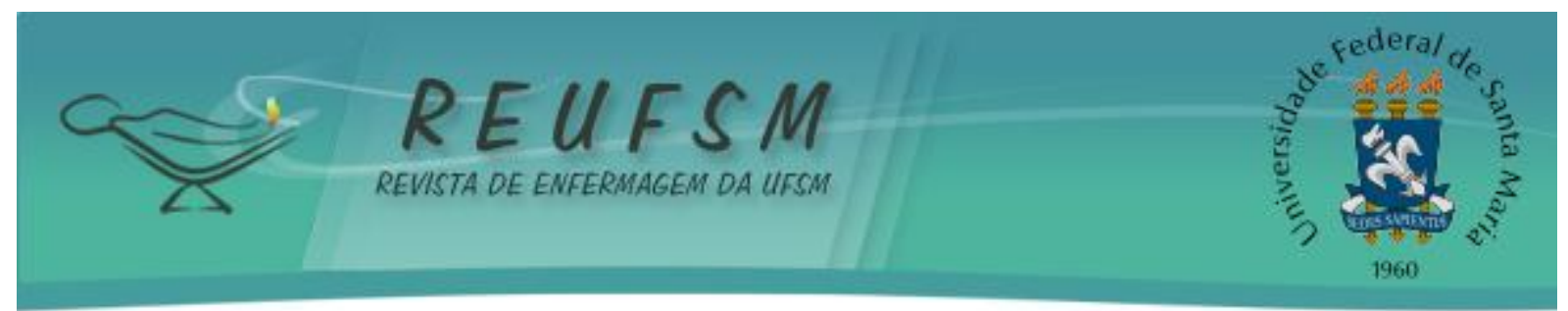

and Family Health Strategy in September from 2009 to July 2010, during the period of supervised trainee in Undergraduate Nursing Courses. The actions compacted in practices of health education in hospital and community spheres. Results: the educational activities were developed in the admission of the user to the service, in group technics and domiciliary visits in order to that they co-responsibilities for its promotion of health, prevention, treatment and recovery of cardiovascular diseases. Conclusion: contrast the need to usehealth education actions in care practices as an element of emancipative users in their self-care.

Descriptors: Health education; Cardiovasculares disease; Nursing care.

RESUMEN: Objetivo: describir las actividades de promoción, prevención, recuperación y tratamiento de las enfermedades cardiovasculares desarrollado en práctica de la atención, junto con los usuarios con enfermedad del corazón o de los pacientes con un factor de riesgo para la enfermedad. Método: se trata de un relato de experiencia obtenida en un Laboratorio de Hemodinámica, la Unidad de Cardiología Intensiva y la Estrategia de Salud Familiar en Septiembre de 2009 hasta Julio de 2010, durante el período de prácticas supervisadas en el Curso de Graduación de Enfermería. Las acciones consistieron en prácticas de educación en salud en el escenario hospitalario y comunitario. Resultados: las actividades educativas fueron desarrollados en la admisión del usuario al servicio, en técnicas grupales y visitas domiciliarias con el fin de que ellos mismos se corresponsabilicen para la promoción de la salud, prevención, tratamiento y recuperación de enfermedades cardiovasculares. Conclusión: es importante destacar la necesidad de emplear medidas de educación en salud en las prácticas de atención como un elemento emancipatorio de los usuarios en sus cuidados personales.

Descriptores: Enfermedades cardiovasculares; Educación en salud; Atención en enfermería.

\section{INTRODUÇÃO}

O cotidiano agitado, a falta de exercícios físicos, o estresse, a alimentação rápida, rica em gordura e açúcar e, sobretudo, o consumo excessivo de bebidas alcoólicas e tabaco predispõem ao aparecimento das doenças cardiovasculares (DCV). Essas doenças são nos dias de hoje, uma preocupação mundial, pois, direta ou indiretamente são responsáveis por um elevado número de mortes em todos os países. ${ }^{1}$

Tal questão ocorre porque apesar do avanço na área cardiovascular por meio do incremento de conhecimentos, desenvolvimento de recursos diagnósticos e terapêuticos, o quadro epidemiológico das DCV ainda é crescente. Diversos são os fatores de risco relacionados, como: obesidade, sedentarismo, hipertensão arterial, diabetes mellitus, alcoolismo, tabagismo, dentre outros. ${ }^{2}$

As DCV são a principal causa de morbidade, incapacidade e morte no mundo e no Brasil, sendo responsáveis por $29 \%$ das mortes registradas em 2007. Os gastos com internações pelo SUS totalizaram 1,2 milhões em 2009 e, com o envelhecimento da população e mudança dos hábitos de vida, a prevalência e importância das DCV tende a aumentar nos próximos anos. ${ }^{3}$

Dentre as DVC que mais acometem a população, destacam-se a Insuficiência Cardíaca e as Cardiopatias Isquêmicas (a doença Arterial Coronariana e o Infarto Agudo do Miocárdio). ${ }^{4}$ Seu aparecimento deve-se a combinação de fatores genéticos, socioeconômicos e ambientais, sendo estes dois últimos resultantes do estilo de vida adotado na fase adulta. ${ }^{5}$

Por conseguinte, a Insuficiência Cardíaca é descrita como uma síndrome clínica, caracterizada pela incapacidade do coração em suprir adequadamente as necessidades 


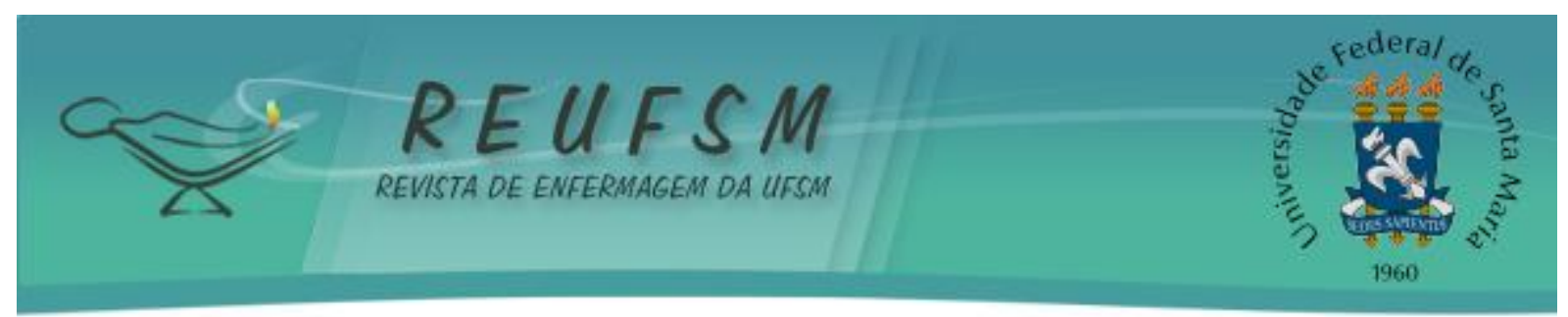

metabólicas do organismo ou fazê-lo sob elevadas pressões de enchimento das câmaras cardíacas. ${ }^{6}$ Segundo dados do Departamento de Informática do Sistema Único de Saúde (DATASUS) ela é a principal causa de hospitalização entre as DVC. ${ }^{7}$

A Cardiopatia Isquêmica é outro sério problema de saúde, que ocorre em consequência do fluxo inadequado de sangue pelas artérias coronárias. Em acréscimo, o mecanismo patológico pré-existente a Cardiopatia Isquêmica, na maioria dos casos, é a obstrução coronariana por placas ateromatosas, ocasionando a aterosclerose, que impedem a chegada de oxigênio e nutrientes ao miocárdio, podendo evoluir para Infarto Agudo do Miocárdio. ${ }^{8}$

Assim, a ocorrência de isquemia intensa e contínua, numa porção do miocárdio pode ocasionar lesão irreversível e morte celular, resultando em infarto. ${ }^{4}$ Segundo dados do DATASUS, ocorreram no Brasil 1.099.131 mortes em 2009, 99.835 foram atribuídas às doenças isquêmicas do coração, das quais 75.868 são decorrentes do infarto agudo do miocárdio, o que representa $6,9 \%$ de todas as mortes. ${ }^{3}$

Isso acarreta diretamente no aumento do custo assistencial de saúde, incluindo os gastos com internação hospitalar, medicamentos, exames complementares, absenteísmo, aposentadoria precoce e consultas ambulatoriais. ${ }^{5}$ Dentre os exames diagnósticos mais precisos destaca-se a Angiografia Coronariana (AC), que avalia a presença e localização da arteriopatia coronariana. ${ }^{4} \mathrm{E}$, dentre os métodos terapêuticos descrevem-se a Angioplastia Coronariana Transluminal Percutânea (ACTP) e a Cirurgia de Re-vascularização do Miocárdio (CRM).

A ACTP consiste na introdução de uma endoprótese arterial (stent), a fim de desobstruir a coronária ocluída e a CRM é um procedimento cirúrgico para realização de desvio em grave estenose, feito com enxerto de veia safena e ou artéria mamária interna, para restabelecer o fluxo sanguíneo ideal naquela região do miocárdio. ${ }^{4}$

Consequentemente, dos avanços diagnósticos e terapêuticos somados a magnitude dos problemas relacionados às doenças cardiovasculares, torna-se prioridade a implementação de estratégias que visem à educação em saúde dos usuários acometidos por tais enfermidades. Isso possibilita o aprimoramento do cuidado individualizado e competente, identificando precocemente os fatores de risco e a instauração de enfermidades cardiovasculares, além de fornecer subsídios ao usuário e a família quanto ao conhecimento de sua doença e cuidados que devem ser adotados.

Diante disso, elaborou-se este relato de experiência, obtido durante o período de estágio supervisionado pelo Curso de Enfermagem, com objetivo de descrever as atividades de promoção, prevenção, recuperação e tratamento de doenças cardiovasculares desenvolvidas na prática assistencial, juntamente com os usuários cardiopatas ou portadores de algum fator de risco à doença.

\section{Percurso e ações na prática assistencial}

As ações educativas e de cuidado foram desenvolvidas com usuários portadores de enfermidades cardiovasculares ou com fatores de risco predisponentes a essas doenças. Tais atividades, executadas por uma concluinte do Curso de Graduação em Enfermagem, ocorreram nos cenários da atenção básica (em uma Estratégia de Saúde da Família - ESF alocada em uma cidade do interior do Rio Grande do Sul) e hospitalar (em um hospital escola da mesma cidade), no período de setembro de 2009 a julho de 2010.

Como estratégia assistencial, desenvolveram-se ações da prática assistencial, no Laboratório de Hemodinâmica, uma participação no grupo de Educação em Saúde a pacientes pré-cirúrgicos de CRM na Unidade de Cardiologia Intensiva (UCI), e também no grupo de hipertensos e diabéticos na ESF, além de atuar junto às Agentes Comunitárias de Saúde (ACS) nas visitas domiciliárias para acompanhamento e implementação de ações de 


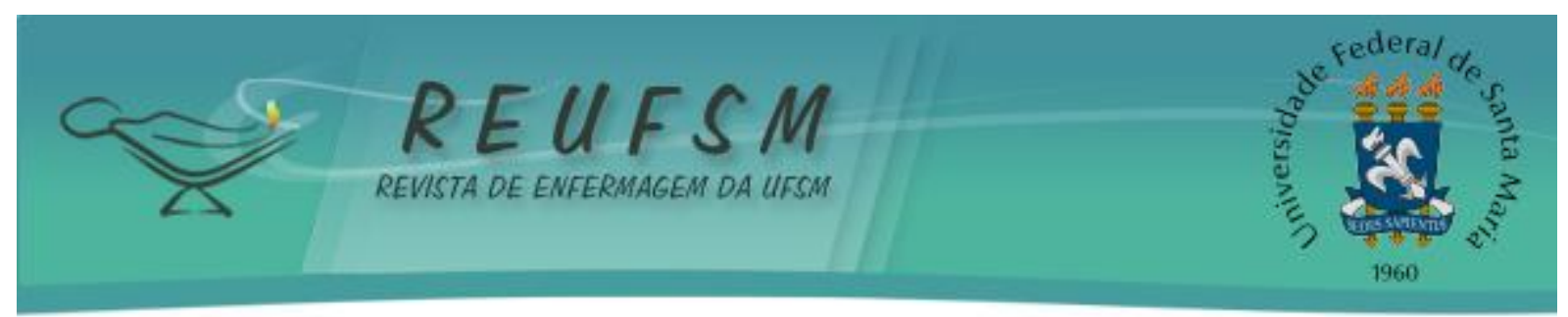

educação em saúde. Destaca-se que era de conhecimento tanto dos usuários como dos profissionais dos serviços, onde a prática assistencial foi executada, que essas atividades seriam divulgadas na forma de relatório de estágio bem como, posteriormente, na forma de relato de experiência.

Essas práticas de educação em saúde são pertinentes, uma vez que, revelam-se como ferramentas facilitadoras ao exercício de reflexão e consciência crítica dos usuários sobre as causas de seus problemas de saúde. Assim, possibilita-se enfatizar o desencadeamento de um processo pautado no diálogo, de modo que se elaborem estratégias de cuidado valorativas à experiência e contexto de cada indivíduo, e ocorra a inserção desse sujeito como co-participe em seu plano de cuidados. ${ }^{9}$

Nessa perspectiva, educar em saúde é parte integrante do cuidar em enfermagem que "envolve múltiplas dimensões de natureza empírica, ética, estética e pessoal, pois, não é uma atividade ou ação isolada". ${ }^{10: 82}$ Por isso, o cuidar/assistir humano permite compreender, estar com o usuário de modo a ajudá-lo e educá-lo, facilitando o alcance de certos objetivos. $^{10}$

O desenvolvimento da prática assistencial no Laboratório de Hemodinâmica ocorreu em três momentos nos turnos da manhã e tarde: na recepção e preparo dos usuários, no acompanhamento aos procedimentos, e durante o período de recuperação. Esse serviço realiza procedimentos cardíacos diagnósticos (AC, ventriculografia, entre outros) e terapêuticos (ACTP e implante de marcapasso) em pacientes ambulatoriais e internos do próprio hospital.

Como rotina do setor, na recepção dos usuários avaliava-se o cumprimento do preparo para efetuação dos procedimentos, por exemplo: suspensão de algumas medicações, jejum e a assinatura do termo de consentimento para a intervenção.

A seguir, era preenchido um formulário, juntamente com os pacientes e na companhia de seus familiares/acompanhantes, para elencar questões importantes, como: presença de alergias, hipertensão arterial ou diabetes, portador ou não de doenças sexualmente transmissíveis, glaucoma, doença de Chagas ou infecção de qualquer natureza, presença de doença renal, tipo de medicação em uso, descrição do tipo da dor que sentiam no peito (destinado aos pacientes submetidos a AC ou ACTP), verificação de exames anteriores recentes (ecocardiograma, hemograma, eletrocardiograma, testes ergométricos, entre outros), laudos de exames diagnósticos e angioplastias anteriores.

Posteriormente, realizava-se o preparo dos usuários para os procedimentos, que consistia em verificação dos sinais vitais, tricotomia, punção venosa periférica e instalação de soroterapia, em casos de ACTP ou implante de marcapasso cardíaco.

$\mathrm{Na}$ sequência, explicava-se aos usuários e seus familiares a finalidade de cada procedimento, assim como a importância que sua realização representaria na evolução ou análise de seu quadro clínico. Essa prática tranquilizava-os no pós-procedimento já que, os mesmos haviam sido esclarecidos, o que facilitou também em sua colaboração na recuperação.

Cabe ressaltar que, para essas ações educativas elaborou-se um pequeno álbum seriado com figuras ilustrativas e legendas explicativas como parte das atividades assistenciais na recepção, recuperação e preparo para alta hospitalar, a fim de facilitar a compreensão dos usuários. Essa prática proporcionou a visualização do processo de formação da coronariopatia e a forma de realização dos procedimentos terapêuticos ou diagnósticos empregados.

Já na sala de recuperação, assistiu-se aos usuários desde a retirada do introdutor arterial, utilizado durante os procedimentos, no gerenciamento do cuidado de enfermagem, até a posterior alta do setor. Essa responsabilidade permitiu autonomia frente às constantes exigências do serviço, bem como garantiu a inserção na rotina do serviço e integração com a equipe de enfermagem e demais profissionais. 


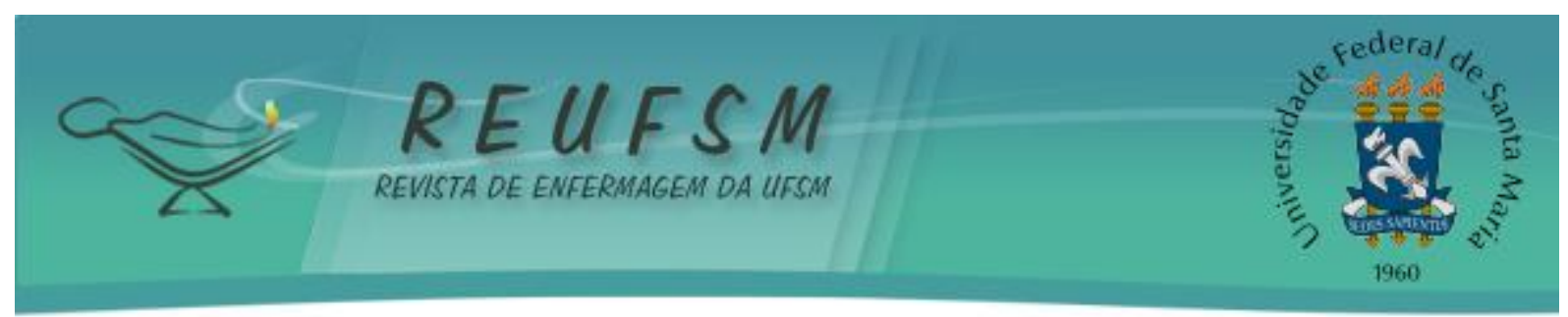

Na saída dos usuários para o seu domicílio foram reforçadas as questões de educação em saúde quanto aos cuidados com o membro utilizado para intervenção no procedimento, estimulação para hábitos saudáveis de vida no que tange alimentação, exercícios físicos regulares, abandono do tabagismo e alcoolismo, administração correta de terapia medicamentosa. Em algumas situações, foram realizadas visitas a pacientes que seriam submetidos a procedimentos cardíacos no setor e que se encontravam internados em outras Unidades do hospital com a finalidade de proporcionar educação em saúde a esses indivíduos.

Quanto à prática assistencial na $\mathrm{UCl}$ participou-se de um grupo de educação em saúde, já existente, com usuários pré-cirúrgicos de CRM antes de serem internados nessa Unidade. Esse serviço atende usuários no pós-operatório de cirurgias cardíacas, emergências cardiológicas, usuários submetidos a estudos hemodinâmicos e que necessitam de monitorização cardíaca. Durante os grupos explicou-se o trans-operatório, desde o momento da indução anestésica, a esternotomia, a retirada de vasos (veia safena ou desvio da artéria mamária) para efetuação da re-vascularização miocárdica, a posterior colocação de drenos, assim como a importância de estar empregando esse tipo de terapêutica.

$\mathrm{Na}$ sequência, informava-se a eles que sua permanência seria na UCl para que tivessem acompanhamento intensivo de seu quadro clínico, reduzindo assim riscos e minimizando danos. Em consequência, percebeu-se que no pós-cirúrgico os usuários e familiares ficaram menos apreensivos e atônitos com os aparelhos, drenos e sondas utilizadas, pois possuíam conhecimento prévio dos acontecimentos subsequentes.

Essa atividade educativa também foi desenvolvida, por meio de visitas, com outros usuários que se encontravam em outras unidades do hospital impossibilitados de participar dos grupos. Nestas visitas eram feitos os mesmos esclarecimentos, porém as atividades educativas ocorriam na enfermaria, onde aguardavam a cirurgia.

Ressalta-se ainda a relevância em desenvolver grupos de educação em saúde na prática do cuidado, pois, eles constituem uma técnica coletiva, entre os sujeitos envolvidos, cujo objetivo visa promover um processo de aprendizagem que favoreça e intensifique as diversas trocas de saberes. ${ }^{11}$

Já no desenvolvimento da prática assistencial, na ESF, foram realizadas visitas domiciliárias aos usuários hipertensos e diabéticos, no intuito de conhecer o contexto em que estavam inseridos, fomentando, assim, a educação em saúde para incentivar a prevenção de agravos, promoção da saúde e manutenção do tratamento.

Em vista disso, a visita domiciliária possibilita à equipe de saúde a observação do indivíduo dentro de seu contexto, meio ambiente, condições de habitação, higiene, saneamento básico e relações afetivo-social entre os membros da família. ${ }^{12}$ Além disso, a proposta da visita domiciliária se torna essencial, pois o profissional ao adentrar no espaço privado da família poderá obter maiores possibilidades de oferecer uma assistência adequada.

A população atendida nesse serviço caracterizava-se por ser de baixo nível socioeconômico, pouca escolaridade e precárias condições de saúde e higiene. Além disso, havia elevada incidência de fatores de risco predisponentes ao aparecimento de enfermidades cardiovasculares, como: hipertensão arterial, diabetes melittus, obesidade, tabagismo, sedentarismo, alcoolismo e a não utilização da terapia medicamentosa.

No esquema semanal, de visitas domiciliárias realizadas juntamente com as ACS, foram aferidos os níveis pressóricos e glicêmicos, sendo que com base nos resultados desenvolveram-se condutas, como: encaminhamento à ESF para consulta médica e posterior avaliação especializada de um cardiologista disponível na rede pública de saúde.

Em acréscimo, efetuaram-se grupos de educação em saúde com usuários hipertensos e diabéticos, em um salão anexo a Unidade, com a parceria de toda equipe de saúde do local. Dentre as atividades desenvolvidas citam-se a discussão sobre prevenção dos fatores de risco das doenças cardíacas, esclarecimentos sobre processos mórbidos que mais acometem a comunidade, 


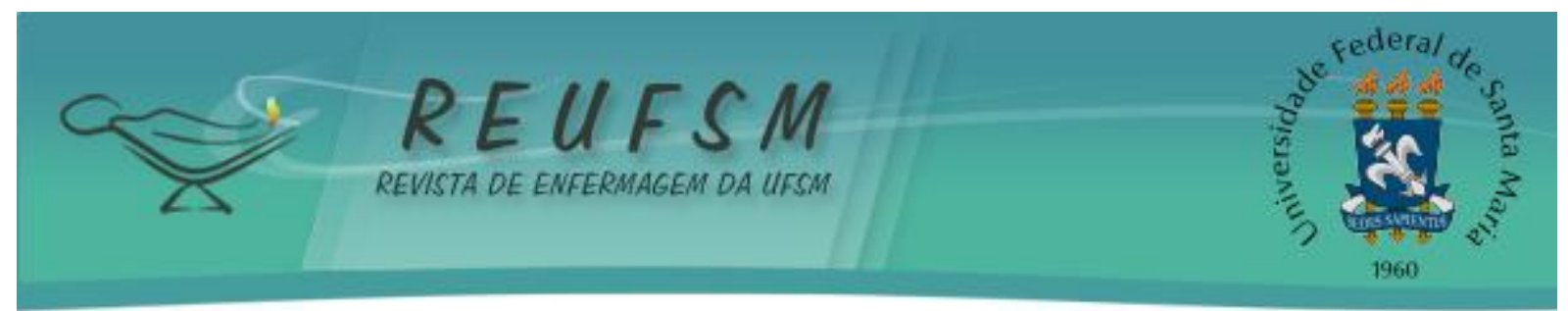

como infarto agudo do miocárdio, hipoglicemia e hiperglicemia. Após, desenvolveu-se uma atividade lúdica, com objetivos claros e coesos, denominada "dança-da-vassoura", a fim de promover entretenimento, descontração e interação entre os sujeitos envolvidos.

No âmbito da atenção básica, resgatou-se, juntamente com os usuários, a importância que a promoção da saúde e prevenção de agravos representa diante da gravidade de uma doença cardiovascular em estágio avançado. Outra questão é que, as visitas domiciliárias e os grupos de educação em saúde sustentam a corresponsabilidade entre usuário e serviço, pois os mesmos devem ser copartícipes no processo do cuidado.

\section{CONSIDERAÇÕES FINAIS}

A vivência dessa prática assistencial potencializou o desenvolvimento de capacidades e ações educativas críticas e reflexivas na práxis do cuidado frente às dificuldades e desafios apresentados na prevenção, controle, recuperação e tratamento das enfermidades cardiovasculares. Para isso, a colaboração e convivência com cada membro das equipes de saúde durante a realização dessa vivência foram determinantes.

Em acréscimo, fomentar a discussão acerca de enfermidades cardiovasculares possibilitou um espaço de identificação e troca de idéias por meio da socialização dos resultados da vivência. Além disso, as considerações expostas descrevem a importância de articular educação em saúde em práticas assistenciais do cuidado, por meio de grupos, no espaço das visitas domiciliárias e na própria admissão do usuário no ambiente hospitalar. Essas trocas do saber permitem interação e integração entre serviço de saúde, profissional e usuário.

A abordagem educativa para o cuidado com o usuário, requer dos profissionais da saúde o emprego de seu tempo, conhecimento, competência e planejamento para melhorar a qualidade da assistência e obter resultados satisfatórios na manutenção ou recuperação da saúde.

\section{REFERÊNCIAS}

1. Santos ALT, Weiss T, Duarte CK, Azevedo MJ, Zelmanovitz T. Análise crítica das recomendações da Associação Americana de Diabetes para doença cardiovascular no diabetes melito. Arq bras endocrinol metab [internet]. 2009 [acesso em $2011 \mathrm{dez}$ 16];53(5):657-66. Disponível em: http://www.scielo.br/ pdf/abem/v53n5/18.pdf.

2. Carvalho LDP, Dias RS, Santos EA, Portella TRA, Pestana RMS. Educação e cuidado cardiovascular: a importância das atividades de promoção e prevenção. In: Anais do $16^{\circ}$ Seminário de Enfermagem em Pesquisa, 2011 jun. 19-22; Campo Grande. Campo Grande: ABEn-Seção-MS; 2011. p. 2316-8.

3. Ministério da Saúde (BR). Departamento de Atenção Especializada. Secretaria de Atenção à Saúde. Decreto $N^{\circ}$ 4.176, de 28 de março de 2002: Linha do cuidado do infarto agudo do miocárdio na rede de atenção às urgências [internet]. 2011 [acesso em 2011 dez 16]. Disponível em: portal.saude.gov.br/portal/arquivos/pdf/cnos ulta_cardio_062011.pdf.

4. Pittan LO, Senger R, Guido LA. Caderno didático: assistência de enfermagem em cardiologia. Santa Maria: Universidade Federal de Santa Maria; 2006.

5. Hudak CM, Gallo BM. Cuidados intensivos de enfermagem: uma abordagem holística. Rio de Janeiro: Guanabara Koogan; 1997.

6. Neto AGC, et al. Reabilitação cardíaca após alta hospitalar no sistema público de saúde do município do Rio de Janeiro. Rev SOCERJ [internet]. 2008 [acesso em 2011 fev 20]; 


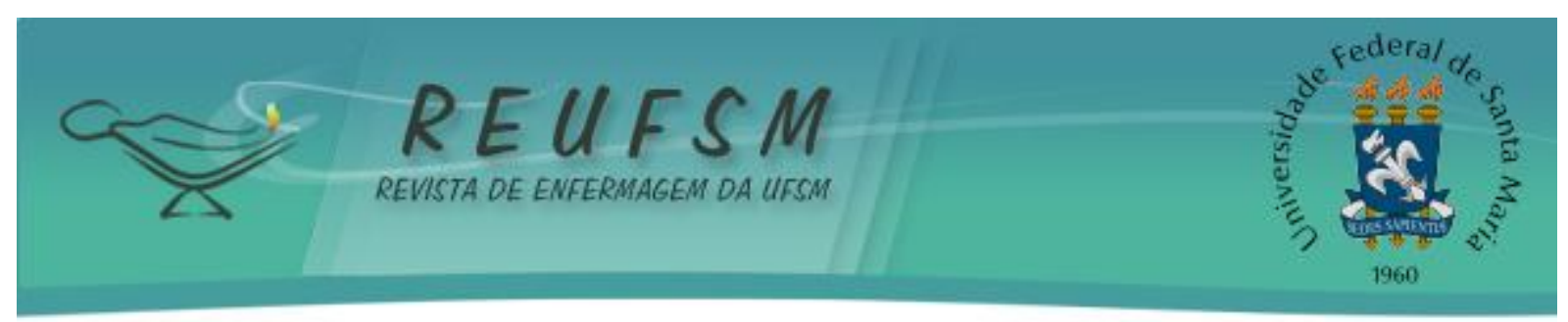

21(6):399-403. Disponível em: http://sociedades.Cardiol.br/socerj/revista/ 2008_06/a2008_v21_n06_a06antoniogilcastinheiras.pdf.

7. Tardin OMA, et al. Estudo de polimorfismos Genéticos na Insuficiência Cardíaca (GenetIC): delineamento do estudo e metodologia. Rev. SOCERJ [internet]. 2009 [acesso em 2011 jan 6];22(1):36-42. Disponível em: http://sociedades.cardiol.br/ socerj/revista/2009_01/a2009_v22_n01_a05Tardin.pdf

8. Knobel E. Terapia intensiva: cardiologia. São Paulo: Atheneu; 2003.

9. Alves GG, Aert AD. As práticas educativas em saúde e a ESF. Ciênc saúde coletiva [internet]. 2011 [acesso em 2011 abr 12];16(1):319-25. Disponível em:

http: / / www.scielosp.org/scielo.php?script=sci_arttext\&pid=S141381232011000100034\&lang=pt.

10. Neves EP. As dimensões do cuidar em enfermagem: concepções teórico-filosóficas. Esc Anna Nery rev enfermagem. 2002;6(1):79-92.

11. Torres HC, Hortale VA, Schal V. A experiência de jogos em grupos operativos na educação em saúde para diabéticos. Cad saúde pública [internet]. 2003 jun/ago [acesso em 2011 abr 12];19(4):1039-47. Disponível em: http://www.scielosp.org/ pdf/csp/v19n4/16853.pdf.

12. Labate RC, Galera SAF, Avanci R. Visita domiciliária: um olhar da enfermagem psiquiátrica. Rev bras enferm. [internet]. 2004 [acesso em 2011 abr 14]; 57(5):627-8. Disponível em: http://www.scielo.br/pdf/reben/v57n5/a24 v57n5.pdf

Data de recebimento: $27 / 04 / 2011$

Data de aceite: 06/03/2012

Contato com autor responsável: Márcia Gabriela Rodrigues de Lima Endereço: Rua Afonso Potter, 2077. Restinga Seca/RS, Brasil.

CEP: $97200-000$

E-mail: grlmarcia@yahoo.com.br 